\title{
Intelligent Experiment Design-Based Virtual Remote Sensing Laboratory
}

\author{
Yuriy Shkvarko, Stewart Santos, and Jose Tuxpan \\ Department of Electrical Engineering, CINVESTAV-IPN, Guadalajara, Mexico \\ \{shkvarko, ssantos, jtuxpan\} @gdl.cinvestav.mx
}

\begin{abstract}
We address unified intelligent descriptive experiment design regularization (DEDR) methodology for computer-aided investigation of new intelligent signal processing (SP) perspectives for collaborative remote sensing (RS) and distributed sensor network ( $\mathrm{SN}$ ) data acquisition, intelligent processing and information fusion. The sophisticated "Virtual RS Laboratory" (VRSL) software elaborated using the proposed DEDR methodology is presented. The VRLS provides the end-user with efficient computational tools to perform numerical simulations of different RS imaging problems. Computer simulation examples are reported to illustrate the usefulness of the elaborated VRSL for the algorithmic-level investigation of high-resolution image formation, enhancement, fusion and post-processing tasks performed with the artificial and real-world RS imagery.
\end{abstract}

Keywords: Computer simulations, experiment design, regularization, remote sensing, software.

\section{Introduction}

This paper is focused on the challenging problems of intelligent remote sensing (RS) data processing, distributed fusion, algorithm design and simulation software development. First, we address a unified intelligent descriptive experiment design regularization (DEDR) methodology for (near) real time formation/enhancement/ reconstruction/post-processing of the RS imagery acquired with different types of sensors, in particular, conventional 2-D stationary arrays [1] and the mobile synthetic aperture radar (SAR) systems [2]. Second, we present the elaborated "Virtual Remote Sensing Laboratory" (VRSL) software that provides the end-user with efficient computational tools to perform numerical simulations of different collaborative RS imaging problems in various experiment design settings. The scientific challenge is to develop and investigate via the VRSL an intelligent signal processing (SP) perspective for collaborative RS data acquisition, adaptive processing and information fusion for the purposes of high-resolution RS imaging, search, discovery, discrimination, mapping and problem-oriented analysis of spatially distributed physical RS signature fields. The end-user oriented VRSL software is elaborated 
directly to assist in system-level and algorithmic-level investigation of such multi-sensor collaborative image formation, enhancement, and post-processing tasks performed with the artificial and real-world RS imagery.

\section{Unified DEDR Paradigm}

The DEDR paradigm constitutes a methodology for solving a manifold of algorithm design problems related to high-resolution multisensor imaging and knowledgebased (KB) collaborative RS data processing. In the DEDR framework developed originally in [8], [10], [11] complex multisensor measurement data wavefields in the observation domain are modeled as operator transforms of the initial scene scattering fields degraded by clutter and noise. The formalism of such transforms is specified by the corresponding uncertain signal formation operator (SFO) models derived from scattering theory [2], [4]. In [8], [10], [11], we followed a generalized maximum entropy (ME) formalization of a priori information regarding the spatial spectrum patterns (SSPs) of the scattered wavefields that unify diverse RS imaging problem settings. Being nonlinear and solution-dependent, the optimal general-form DEDR estimators of the SSPs constructed in [8], [10] require computationally intensive adaptive signal processing operations that involve also the proper construction of the regularizing projections onto convex (solution) sets (POCS) ruled by the adopted fixed-point contractive iteration process. The fused KB DEDR algorithm design methodology [11] aggregates next the ME method with the diverse regularization and KB post-processing considerations. Such the methodology [11] enables one not only to form the atlas of the desired remote sensing signatures (RSS) extracted from the collaboratively processed multisensor RS imagery but also to perform their problem-oriented analysis in an intelligent KB fashion. Figure 1 presents the block-diagram of the addressed intelligent DEDR approach with the KB multisensor data fusion.

\section{DEDR Phenomenology}

Following [6], [8], [10] the DEDR is addressed to as a methodology that unifies the family of the previously developed nonparametric high-resolution RS imaging techniques. Such unified formalism allows involving into the DEDR different convex regularization and neural computation paradigms (e.g., the POCS regularization and $\mathrm{KB}$ method fusion) that enables the end user to modify the existing techniques via incorporation of some controllable algorithmic-level "degrees of freedom" as well as design a variety of efficient aggregated/fused data/image processing methods. The data/image processing tasks that may be performed applying the DEDR methodology can be mathematically formalized in the terms of the following unified optimization problem [8], [10]. 


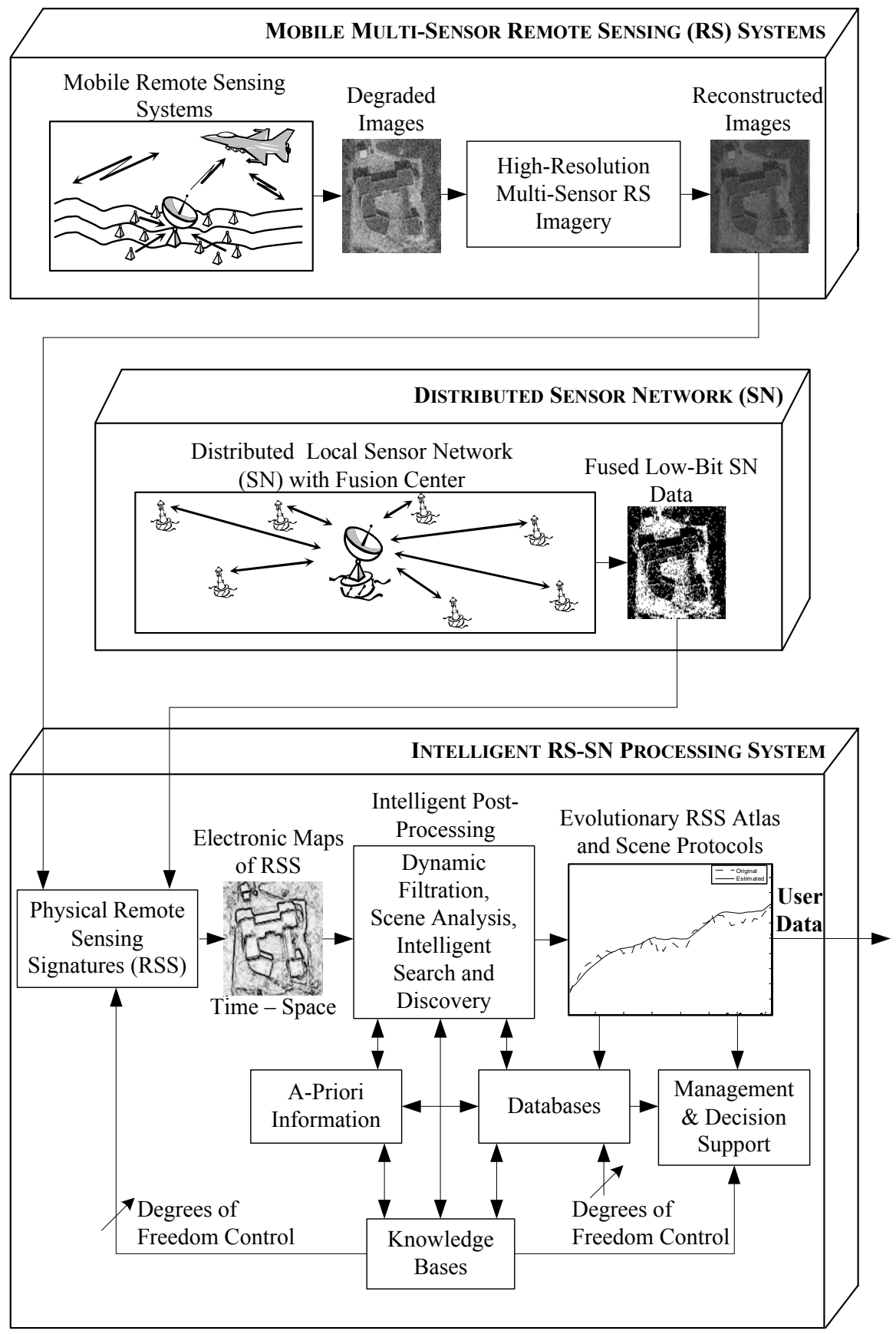

Fig. 1. Block-diagram of the proposed intelligent DEDR approach with KB multisensor data fusion 


$$
\hat{\mathbf{v}}=\underset{\mathbf{v}}{\arg \min } E(\mathbf{v} \mid \lambda)
$$

of minimization of the aggregated objective (cost) function

$$
E(\mathbf{v} \mid \lambda)=-H(\mathbf{v})+(1 / 2) \sum_{m=1}^{M} \lambda_{m} J_{m}(\mathbf{v})+(1 / 2) \lambda_{M+1} J_{M+1}(\mathbf{v})
$$

with respect to the desired $K$-D image vector $\mathbf{v}$ for the assigned (or adjusted) values of $M+1$ regularization parameters $\left\{\lambda_{m}\right\}$ that compose a vector of the controllable algorithmic "degrees of freedom" $\lambda$. In a particular employed method, the proper selection of $\left\{\lambda_{m}\right\}$ is associated with the parametric-level adjustment of the SP optimization procedure (2). Here, $H(\mathbf{v})=-\sum_{k=1}^{K} v_{k} \ln v_{k}$ represents the image entropy [3], $\left\{J_{m}(\mathbf{v})\right\}(m$ $=1, \ldots, M)$ compose a set of particular objective (cost) functions incorporated into the optimization, and $J_{M+1}(\mathbf{v})$ represents the additional regularizing stabilizer [3] that controls specific metrics properties of the desired image. The data acquisition model is defined by the set of equations, $\mathbf{u}^{(m)}=\mathbf{F}^{(m)} \mathbf{v}+\mathbf{n}^{(m)}$ for $M$ methods/systems to be aggregated/fused, i.e. $m=1, \ldots, M$, where $\mathbf{F}^{(m)}$ represent the system/method degradation operators usually referred to as the imaging system point spread functions (PSF), and vectors $\mathbf{n}^{(m)}$ represent composite noises (usually with unknown statistics) in the actually acquired images, respectively.

Different RS imaging methods incorporate different definitions for corresponding employed objective (cost) functions $\left\{J_{m}(\mathbf{v})\right\}$ [2], [3], [5], [6], [8], [10]. For the deterministic constrained least squares (CLS) method [2], [3], $J_{m}(\mathbf{v})=\left\|\mathbf{u}^{(m)}-\mathbf{F}^{(m)} \mathbf{v}\right\|^{2}$, are associated with partial error functions. For the weighted CLS (WCLS) method [6], the objective costs incorporate the user-defined weight matrices $\left\{\mathbf{W}_{m}\right\}$ as additional "degrees of freedom", i.e. $J_{m}(\mathbf{v})=\left\|\mathbf{u}^{(m)}-\mathbf{F}^{(m)} \mathbf{v}\right\|^{2}{ }_{\mathbf{W} m}$. The unified DEDR paradigm incorporates into the unified optimization problem (1), (2) also other robust and more sophisticated statistical methods, among them are: the rough conventional matched spatial filtering (MSF) approach [8]; the descriptive maximum entropy (ME) technique [6]; the robust spatial filtering (RSF) method [5], the robust adaptive spatial filtering (RASF) technique [8], the fused Bayesian-DEDR regularization (FBR) method [5], the POCS-regularized DEDR method, i.e., the unified DEDR-POCS [10]; etc. All such methods involve particular specifications of the corresponding $\left\{J_{m}(\mathbf{v})\right\}$ and $\left\{\mathbf{W}_{m}\right\}$ into the DEDR optimization procedure (1), (2). It is important to note that due to the non-linearity of the objective function (2) the solution of the parametrically controlled fusion-optimization problem (1), (2) will require extremely complex (NPcomplex [10]) algorithms and result in the technically intractable computational schemes if solve these problems employing the standard direct minimization techniques [1], [3]. For this reason, we propose to apply the POCS-regularized fixed-point iterative techniques implemented using the neural network (NN) based computing for solving such aggregated DEDR optimization problems (1), (2) as those were detailed in the previous studies [3], [10], [11]. 


\section{Integrated VRSL Software}

Having developed a manifold of the DEDR-POCS computational techniques, the next goal is to computationally implement, verify, and demonstrate the capabilities of the collaborative RS signal and image processing for RSS extraction, KB intelligent scene analysis, multiple target detection and scene zones localization via development of the sophisticated end-user-oriented software that we refer to as "Virtual remote sensing laboratory" (VRSL). The purpose of the elaborated VRSL software is to implement computationally all considered DEDR-related methods (MSF, CLS, WCLS, ME, RSF, RASF, FBR, etc) and to perform the RS image formation/ reconstruction/enhancement tasks with or without method and/or sensor system fusion. The VRSL software (created in the MATLAB V.7 computational environment) aggregates interactive computational tools that offer to the user different options of acquisition and processing of any image in the JPEG, TIFF, BMP and PNG formats as test input images, application of different system-level effects of image degradation with a particular simulated RS system, simulation of random noising effects with different noise intensities and distributions. Next, various RS image enhancement/fusion/reconstruction/post-processing tasks can be simulated in an interactive mode applying different DEDR-related algorithms to the degraded noised images, and the quantitative performance enhancement characteristics attained in every particular simulated scenario can then be computed and archived [11].

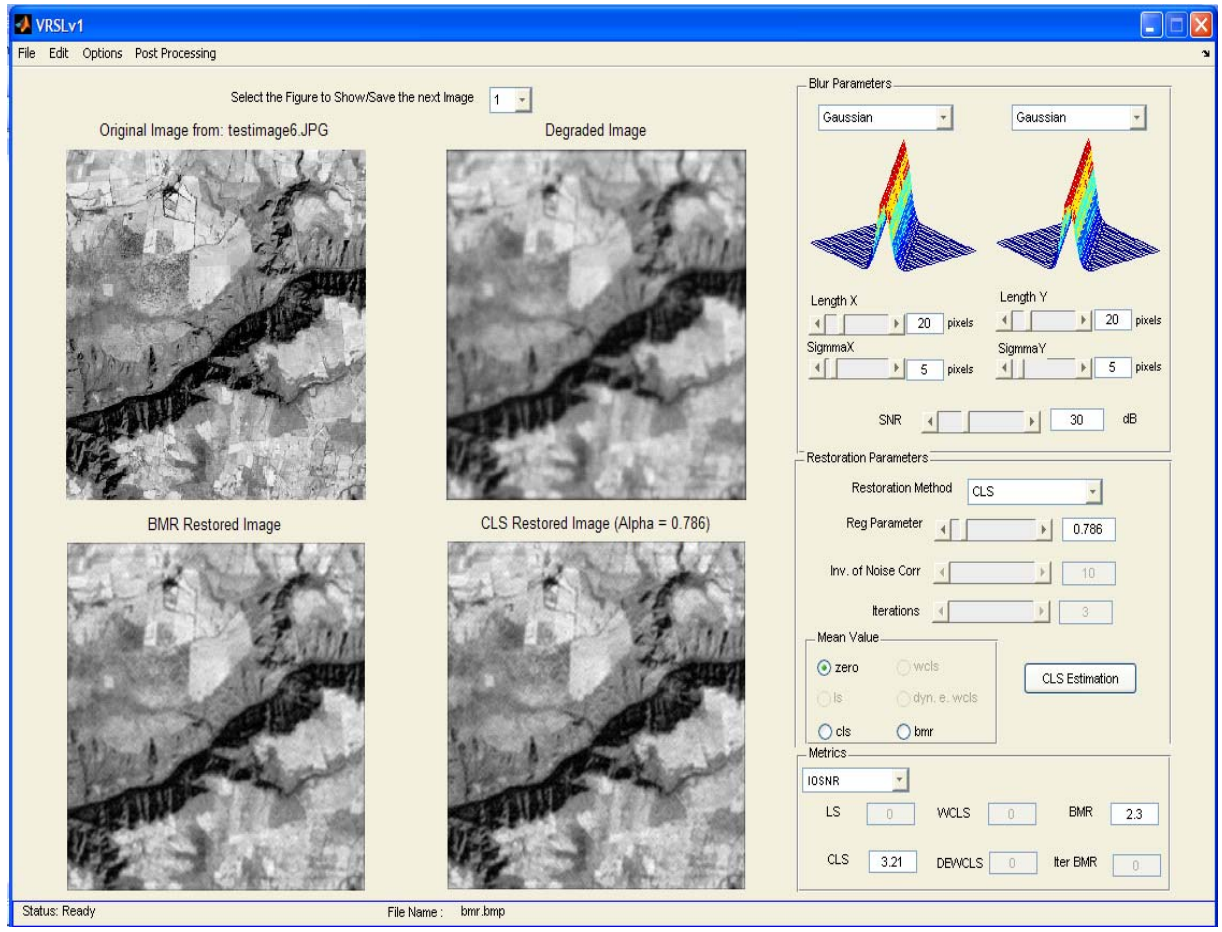

Fig. 2. Elaborated graphical user interface of the VRSL 
The user has options to display on the screen all simulated processed scene images and RSS along with the corresponding protocols of analysis of different performance quality metrics (see the illustrative RS image reconstruction examples displayed in the user interface presented in Fig. 2).

\section{Simulation Examples}

Here, we report some simulations of the DEDR-related algorithms (performed using the elaborated VRSL) carried out in two dimensions for the uncertain operational scenario with the randomly perturbed SFO, in which case the measurement data are contaminated by the composite speckle and multiplicative signal-dependent noise [9], [10]. The simulation experiments that we report in this paper relate to enhancement of the RS images acquired with a fractional SAR system characterized by the PSF of a Gaussian "bell" shape in both directions of the 2-D scene (in particular, of 16 pixel width at 0.5 from its maximum for the 512 -by-512 pixel-formatted test scenes). The chi-squared additive noise with the $\mathrm{SNR}=15 \mathrm{~dB}$ was incorporated to test and compare the performances of different employed enhancement methods. Two scenes (the artificially synthesized and borrowed from the real-world RS imagery) were tested. These are displayed in Figures 3(a) and 3(b), respectively. The qualitative simulation results for six different DEDR-related enhancement/reconstruction procedures for the first test scene are presented in Fig. 4 and for the second scene in Fig. 5, respectively, with the corresponding IOSNR (improvement in the output signal-to-noise ratio [8]) quantitative performance enhancement metrics reported in the figure captions (in the [dB] scale).

From the reported simulation results, the advantage of the well designed imaging experiments (POCS-regularized DEDR, ASF and adaptive RASF) over the case of badly designed experiment (non-robust MSF, de-speckling without DEDR enhancement and non-constrained RSF) is evident for both scenes. Due to the performed regularized inversions, the resolution was substantially improved in both tested scenarios. The higher values of IOSNR were obtained with the adaptive robust DEDR-related estimators, i.e. with the POCS-regularized iterative fixed-point DEDR technique empirically adapted to the uncertain operational scenario.

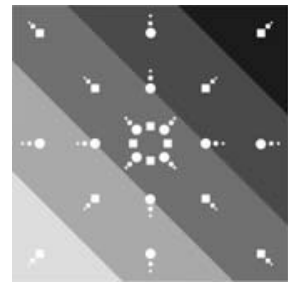

(a)

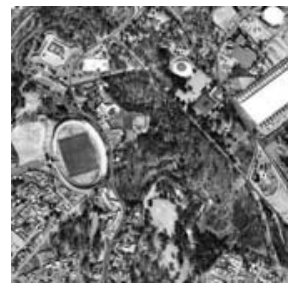

(b)

Fig. 3. Original test scenes: (a) artificially synthesyzed scene; (b) real-world RS scene borrowed from the high-resolution RS imagery [12]. These test scenes are not observable with the simulated SAR imaging system that employs the conventional MSF image formation method. 


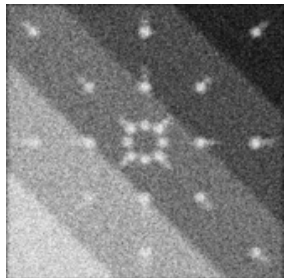

(a)

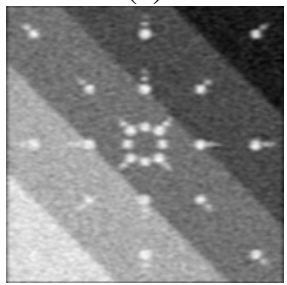

(d)

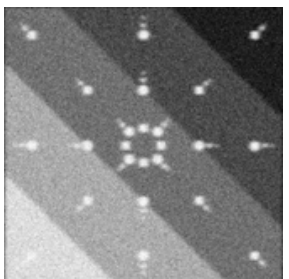

(b)

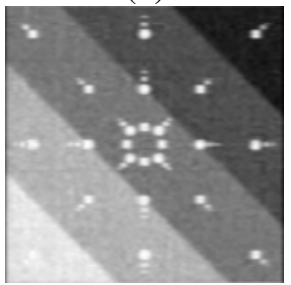

(e)

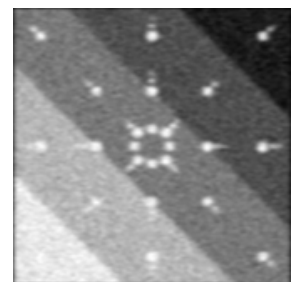

(c)

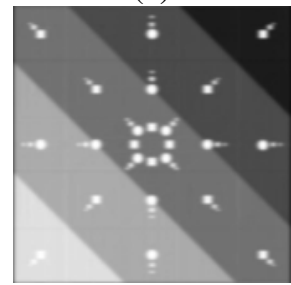

(f)

Fig. 4. Simulation results for the artificially synthesized scene: (a) degraded SAR scene image formed applying the MSF method $[I O S N R=0 \mathrm{~dB}]$; (b) adaptively de-speckled MSF image $[I O S N R=0.62 \mathrm{~dB}]$; (c) image reconstructed applying the non-constrained RSF algorithm $[I O S N R=7.24 \mathrm{~dB}]$; (d) image reconstructed with the constrained RSF algorithm [IOSNR = $8.35 \mathrm{~dB}$ ]; (e) image reconstructed applying the non-constrained ASF algorithm $[$ IOSNR $=9.41$ $\mathrm{dB}]$; (f) image reconstructed applying the POCS-regularized adaptive DEDR method [IOSNR = $15.70 \mathrm{~dB}$ ]

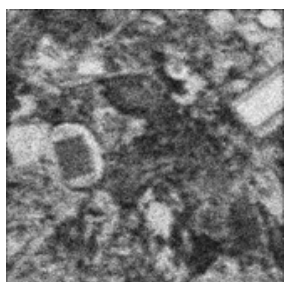

(a)

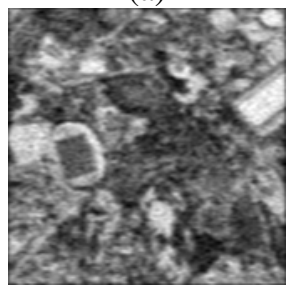

(d)

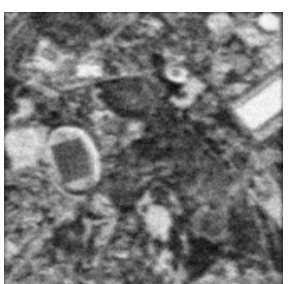

(b)

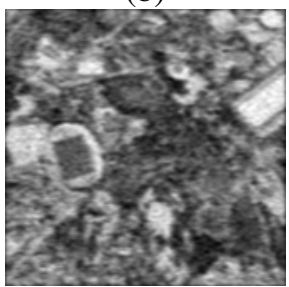

(e)

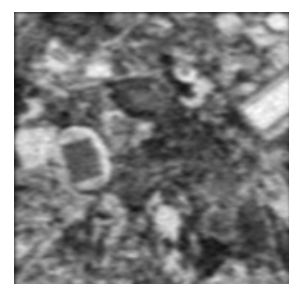

(c)

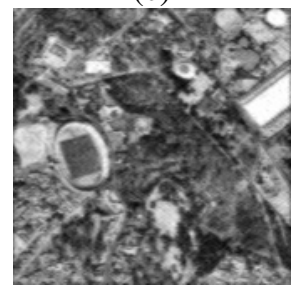

(f)

Fig. 5. Simulation results for the real-world RS test scene: (a) degraded SAR scene image formed applying the MSF method $[I O S N R=0 \mathrm{~dB}]$; (b) adaptively de-speckled MSF image $[I O S N R=0.62 \mathrm{~dB}] ;$ (c) image reconstructed applying the non-constrained RSF algorithm $[I O S N R=6.33 \mathrm{~dB}]$; (d) image reconstructed with the constrained RSF algorithm [IOSNR = $7.78 \mathrm{~dB}]$; (e) image reconstructed applying the non-constrained ASF algorithm $[$ IOSNR $=9.14$ $\mathrm{dB}]$; (f) image reconstructed applying the POCS-regularized adaptive DEDR method [IOSNR = $14.33 \mathrm{~dB}$ ] 


\section{Concluding Remarks}

The descriptive experiment design regularization (DEDR) approach for highresolution estimation of spatial RS signature fields has been unified with the KB post-processing paradigm for the purposes of high-resolution RS imaging, search, discovery, discrimination, mapping and problem-oriented analysis of the diverse RS data. To accomplish computationally different DEDR-specified numerical optimization and processing tasks we have elaborated and reported the end-user-oriented VRSL software. The VRSL provides the necessary tools for computer-aided simulation and analysis of different DEDR-related RS image formation/enhancement/ reconstruction/fusion/post-processing techniques developed using the unified KB DEDR methodology. The reported simulation results are illustrative of the VRSL usefulness and capabilities in computer simulations of different RS imaging tasks performed with the artificial and real-world RS imagery.

\section{References}

1. Falkovich, S.E., Ponomaryov, V.I., Shkvarko, Y.V.: Optimal Reception of Space-Time Signals in Channels With Scattering (in Russian). Radio i Sviaz, Moscow (1989)

2. Wehner, D.R.: High-Resolution Radar, 2nd edn. Artech House, Boston (1994)

3. Barrett, H.H., Myers, K.J.: Foundations of Image Science. Willey, New York (2004)

4. Ishimary, A.: Wave Propagation and Scattering in Random Media. IEEE Press, NY (1997)

5. Shkvarko, Y.: Estimation of wavefield power distribution in the remotely sensed environment: Bayesian maximum entropy approach. IEEE Trans. Signal Proc. 50(9), 2333-2346 (2002)

6. Shkvarko, Y.: Unifying regularization and Bayesian estimation methods for enhanced imaging with remotely sensed data-Part I: Theory. IEEE Trans. Geoscience and Remote Sensing 42(5), 923-931 (2004)

7. Shkvarko, Y.: Unifying regularization and Bayesian estimation methods for enhanced imaging with remotely sensed data-Part II: Implementation and performance issues. IEEE Trans. Geoscience and Remote Sensing 42(5), 932-940 (2004)

8. Shkvarko, Y.: From matched spatial filtering towards the fused statistical descriptive regularization method for enhanced radar imaging. EURASIP J. Applied Signal Processing 2006, 1-9 (2006) Article ID 39657

9. Greco, M.S., Gini, F.: Statistical analysis of high-resolution SAR ground clutter data. IEEE Trans. Geoscience and Remote Sensing 45(3), 566-575 (2007)

10. Shkvarko, Y., Perez-Meana, H., Castillo-Atoche, A.: Enhanced radar imaging in uncertain environment: A descriptive experiment design regularization approach. Int. J. Navigation and Observation 2008, 1-11 (2008) Article ID 810816

11. Shkvarko, Y., Gutierrez-Rosas, J.A., de Guerrero Diaz Leon, L.G.: Towards the virtual remote sensing laboratory: Simulation software for intelligent post-processing of large scale remote sensing imagery. In: Proc. IEEE Intern. Symposium on Geoscience and Remote Sensing, IGARSS 2007, Barcelona, Spain, pp. 1561-1564 (2007)

12. Space Imaging, GeoEye Inc. (2009), http: / / www . spaceimaging . com/quicklook 